\title{
CONSTRUCTION AND TESTING OF A 21 GHZ CERAMIC BASED POWER EXTRACTOR*
}

\author{
D. Newsham, A. Smirnov, D. Yu, DULY Research Inc, Rancho Palos Verdes, CA 90275 \\ W. Gai, R. Konecny, W. Liu, ANL, Argonne, IL \\ H. Braun, G. Carron, S. Doebert, L. Thorndahl, I. Wilson, W. Wuensch \\ CERN, Geneva, Switzerland
}

\begin{abstract}
A ceramic based power extractor [1] operating at $21 \mathrm{GHz}$ was built by DULY Research Inc. and tested at CTF2, the CERN Linear Collider (CLIC) Test Facility. The structure includes a ceramic extractor section, a 2output-port, circular-to-rectangular waveguide coupler, and a 3-port rectangular waveguide combiner that provides for a single output waveguide. Results of cold tests and full beam tests are presented and compared with theoretical and numerical models.
\end{abstract}

\section{INTRODUCTION}

The dielectric based rf power extractor [2] shows great promise for use in a power source of a two-beam collider, having superior performance and a simpler design when compared to conventional high-power sources. As a first test of the power that can be extracted at high frequency from a dielectric structure, DULY Research has manufactured a 21-GHz ceramic power source [1] which was full-beam tested at CTF2. Data was taken for both single-bunch and multi-bunch train operation.

Because of the strict time constraints imposed by the impending shutdown of CTF2, cold test measurements were performed at CERN and ANL after the full beam test was performed. Based on the data from the full-beam experiment along with the measured $\mathrm{S}_{21}$ from the cold test, the peak power produced by the power extractor with a $275 \mathrm{nC}$ bunch train is estimated to be $\sim 50 \mathrm{MW}$, and is consistent with theory.

\section{FABRICATION}

Figure 1 shows a 3D cut-away model of the power extractor with the primary sections split open indicating the internal structure of the separate pieces. The inner diameter of the ceramic tube was $10 \mathrm{~mm}$, and was a compromise between the size of the electron beam ( $\geq 6 \mathrm{~mm}$ ), and the thickness of the wall of the ceramic tube $(1.465 \mathrm{~mm})$. The full length of the ceramic section was $33 \mathrm{~cm}$ and consisted of 4 ceramic tubes. Three of the tubes were $9 \mathrm{~cm}$ each and the final tube was tapered to minimize reflection from the end.

The extractor was constructed of OFHC copper and brazed to two NW40 flanges for mating to the coupler and to the CTF2 beam line. After brazing, the center hole was precision honed such that the ceramic tubes just slipped through without binding. The ceramic was held in place

\footnotetext{
*Work supported by DOE SBIR grant no. DE-FG03-01ER83232.
}

by copper spacers that are part of the flange connections. The waveguide side slots in the coupler and WR42 flange pieces were EDM cut. The assembly was brazed to form a choke joint in the central hole at the downstream side of the coupler, which includes 2 output waveguides and proper flanges for attachment to the extractor and the CTF2 beam line. The WR42 waveguides between the coupler and the combiner were bent and cold tested for equal phase length at CERN and shipped to DULY for brazing to the flanges. The combiner was constructed from two pieces of $304 \mathrm{SST}$. Two $90^{\circ}$-arc grooves were machined into one of the pieces. The slots were plated with copper, and then the $2^{\text {nd }}$ SST piece was brazed on top of the grooved piece to close the waveguide. After brazing, the required flange faces were machined.

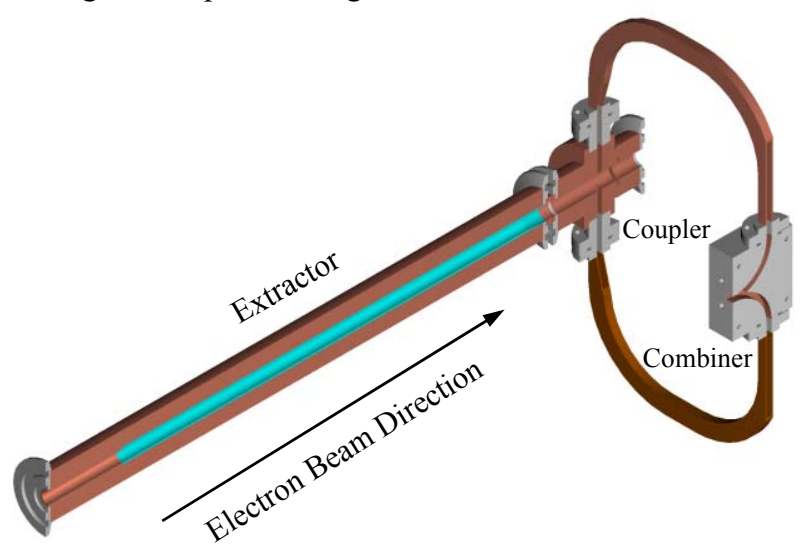

Figure 1: $3 \mathrm{D}$ split model of the $21 \mathrm{GHz}$ ceramic based $\mathrm{rf}$ power extractor.

\section{THEORETICAL MODEL}

The theoretical model [1] for the Cherenkov radiation produced in the ceramic by the passing electron beam was modified to include the effects of reflections at both ends of the ceramic lined tube. This model assumed that there were no reflections at the interfaces between the individual ceramic tubes, that there was no mode conversion within the structure (the coupler could easily cause mode conversion), and that the reflection coefficients at the two ends were constant over the range of study in both time and frequency. Reflections induced by the coupler, choke, or combiner were assumed to be part of the reflection at the end of the extractor tube. The circulating power stored in the cavity is given by:

$$
P_{\text {Intra }}(t) \approx\left|\frac{1-\left(\Gamma_{0} \Gamma_{L}\right)^{1+\left[t / 2 t_{f}\right]}}{1-\Gamma_{0} \Gamma_{L}}\right|^{2} P_{1},
$$


where $t_{f}$ is the filling time, $\Gamma_{0}$ and $\Gamma_{\mathrm{L}}$ are the complex reflections at the beginning and end of the ceramic tube, and $P_{1}$ is the power generated by the ceramic in the absence of any reflections [1]. Two typical cases of Eq. (1) correspond to destructive and constructive interference. Destructive interference occurs for $\mid 1$ $\Gamma_{0} \Gamma_{\mathrm{L}} \mid>1$ and the power circulating in the cavity is identical to the power generated by the electron beam $\mathrm{P}_{1}$. This condition occurs over a wide phase range: $\left|\arg \left(\Gamma_{0} \Gamma_{\mathrm{L}}\right)\right|>\operatorname{Arccos}\left(\left|\Gamma_{0} \Gamma_{\mathrm{L}}\right| / 2\right)$. Constructive interference occurs when $\left|1-\Gamma_{0} \Gamma_{\mathrm{L}}\right|<1$, and the power will build up during the pulse, and the peak power circulating in the cavity will occur when the reflection phase is approximately zero, $\arg \left(\Gamma_{0} \Gamma_{\mathrm{L}}\right) \approx 0$. From 3D electromagnetic simulations using CST Microwave Studio, the reflection at the beam entrance is $\left|\Gamma_{0}\right|=0.992$.

Ultimately, the power that exits the structure is delivered to the load at the power combiner and is determined by $\mathrm{S}_{21}$ assuming no losses in the cavity and the power only exits out the combined port:

$$
P_{\text {Out }} \cong S_{21}^{2} P_{\text {Intra }} \text {. }
$$

\section{COLD TESTS}

Cold tests of the power extractor were performed at CERN and ANL after the full beam tests. Early on during the beam tests, it was noticed that strong reflections were taking place within the structure. A circular mode launcher was designed and constructed at CERN specifically for launching/receiving the test signal at the beginning of the ceramic pieces, and a second launcher was built to match the entrance of the coupler. In addition to the circular launchers, absorbing loads and a single WR42 high power load were used for the cold tests.

A measurement launching from the beginning of the first ceramic tube and ending at the combiner output waveguide resulted in a transmission coefficient: $S_{11}=-12 \mathrm{~dB}$ (ANL measured $-14 \mathrm{~dB}$ ). This number will be used with the measured rf output power in the full beam test to determine the power generated in the extractor.

Subsequently, the coupler was tested separately. With the signal launched at the upstream beam opening, the insertion loss to one of the output flanges (with an absorber in the other one) was $-12.5 \mathrm{~dB}$, not the $-3 \mathrm{~dB}$ that was expected. Finally, the phase difference between the two WR42 output ports on the coupler was $120^{\circ}$ instead of the $180^{\circ}$ required for correct operation of the power combiner. This mismatch could potentially be used to advantage by trapping more power than generated in the extractor (with constructive interference) to test the ceramics breakdown.

\section{FULL BEAM TESTS}

Full beam tests were performed at CERN on CTF2 during the first week in October 2002. Both a single electron bunch and multi-bunch electron trains were used. In all cases, the output rf signal was mixed with a local oscillator $\left(f_{L O}\right)$ and displayed on a digital oscilloscope, where both down-mixed time signal and the FFT spectrum of that signal were displayed. Based on the value of the attenuators, the power calibration (on the time scale display) was $35 \mathrm{~kW}$ for a $0.1 \mathrm{~V}$ (zero-to-peak) signal. The charge of the electron bunches varied (2.5 $5.7 \mathrm{nC}$ per bunch), but the bunch length was between 5 and 6 ps FWHM, and assumed Gaussian.

\section{Single Electron Bunch}

Single bunch experiments were first performed using a bunch charge up to $5 \mathrm{nc}$. Figure 2 shows the results from a single electron bunch test. Figure 3 shows the results of the theoretical model with similar input parameters and numerically mixed with a local oscillator at $23 \mathrm{GHz}$, the same as the experimental data.

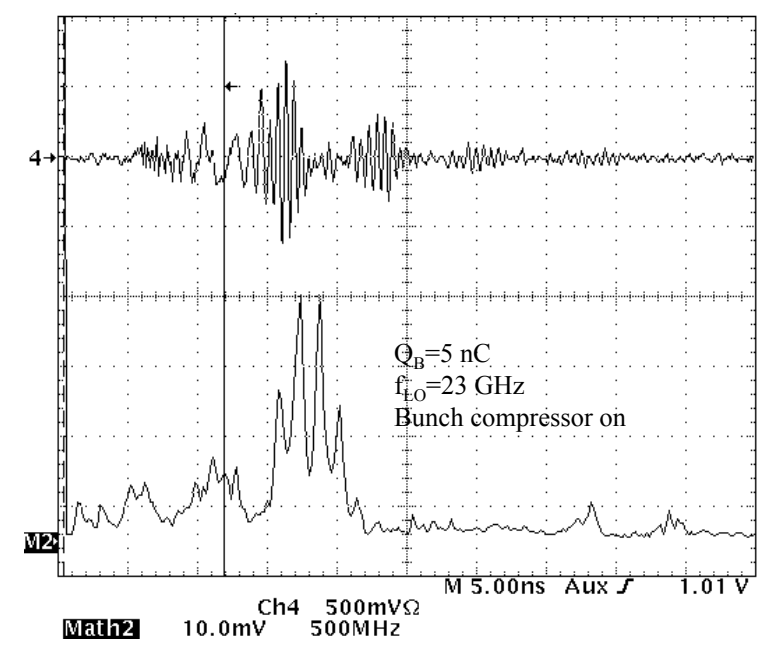

Figure 2: Experimental results of the CTF2 single bunch test. The upper trace is the mixed output time signal; the lower trace is the frequency spectrum of the time signal.

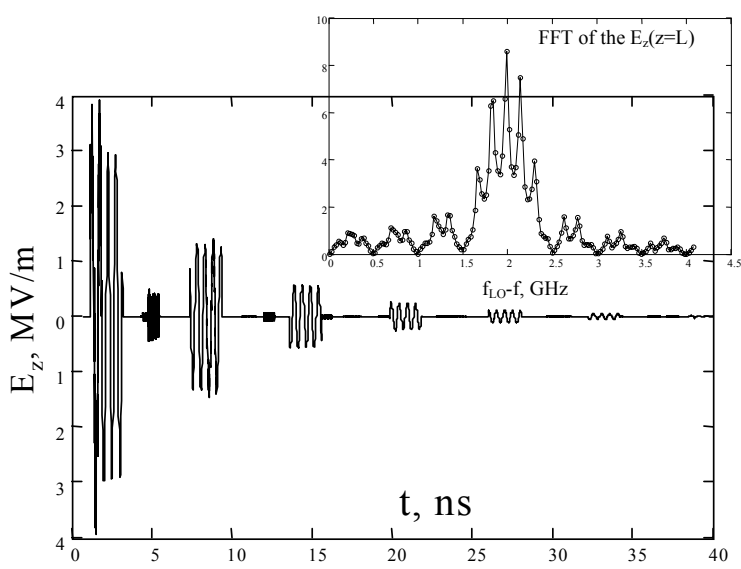

Figure 3: Simulated extractor signal for a single electron bunch mixed with a local oscillator time signal. The insert is the FFT spectrum of the simulated time signal.

Both the experimental and theoretical data for single bunch excitation show the following spectral characteristics:

- The main peak is split into several peaks spaced by approximately $0.16 \mathrm{GHz}$, which corresponds to 
approximately the inverse of twice the filling time, caused by reflections within the length of the ceramic structure.

- $\quad$ Large satellite peaks separated from the main peak by approximately $0.5 \mathrm{GHz}$, which correspond to the inverse of the rf pulse length of the signal produced by a single bunch (drain time).

\section{Multiple Bunch Train}

Electron bunch trains consisting of 48 bunches per macro pulse were the primary focus of study at CFT2. The results were studied for several values of the total charge. Because higher charge resulted in an electron beam that was larger than the beam aperture, multi-bunch operation was functionally limited to a total charge of $275 \mathrm{nC}$, or, $5.7 \mathrm{nC} /$ bunch. Radioactivity measurements after the beam test corroborated that there was beam interception at the entrance of or inside the power extractor tube. The bunch spacing of $0.33 \mathrm{~ns}$ was based on the linac frequency $(3 \mathrm{GHz})$, and the extractor was designed to operate at 7 times the linac frequency. Figure 4 shows a sample experimental time signal and frequency spectrum.

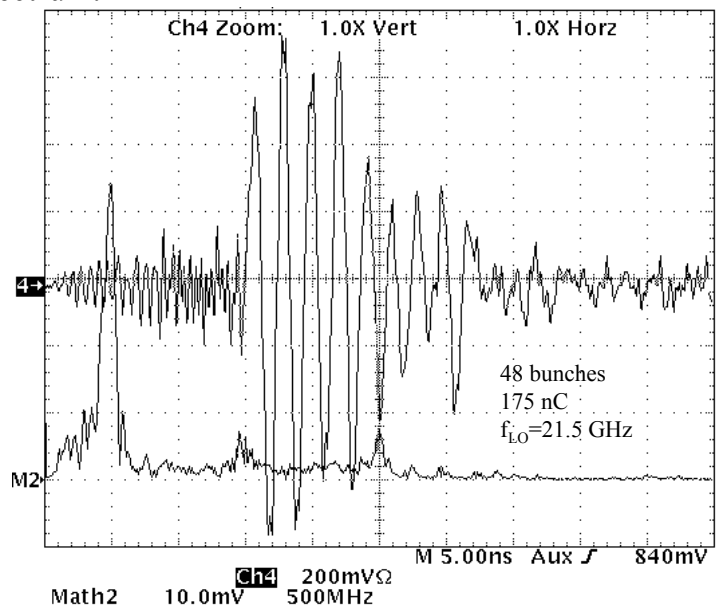

Figure 4: CTF2 results for a multi-bunch electron train.

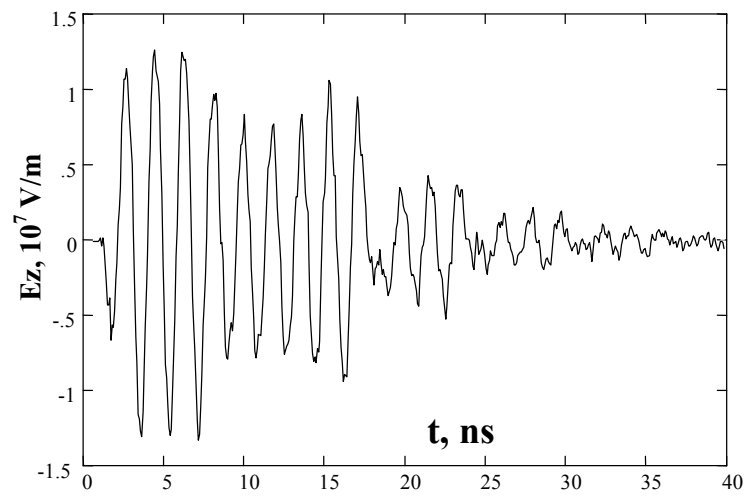

Figure 5: Simulated time signal for the case shown in Figure 4 (different time scale).

Figures 5 and 6 show the analytical calculations for a bunch train with a total charge that is the same as the experimental data, with a local oscillator frequency is $21.5 \mathrm{GHz}$, the same as the experiment.
The results of the cold test data gave a transmission loss of $-12 \mathrm{~dB}$, thus the power generated in the cavity can be calculated by inverting Equation 2: $P_{\text {Intra }} \cong 15.8 P_{\text {out }}$. The apparently low power output is a result of the poor output coupling and is not an indication of low power generation. Figure 7 shows a summary of all the experimental data taken for the multiple bunch train measurements at CTF2 along with a theoretical curve [1] that contains no free parameters. Endoscopic examination after the beam testing revealed signs of damage in the last ceramic tube.

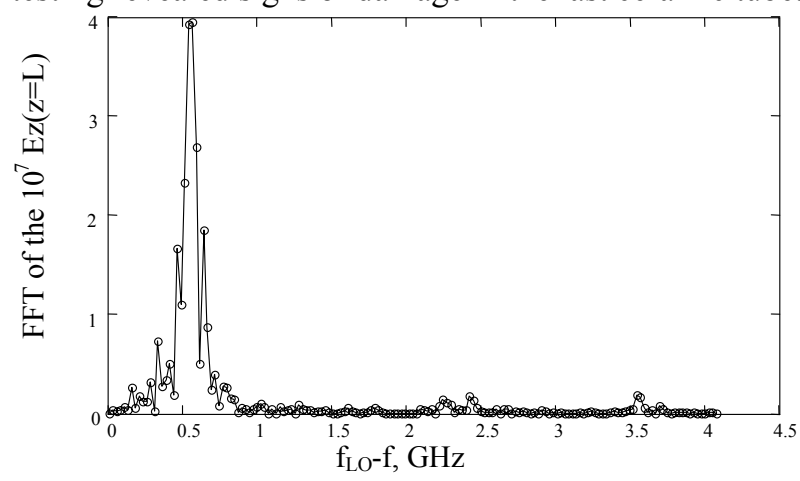

Figure 6: FFT of the simulated time signal shown in Figure 5 (see Figure 4 for comparison with measurement).

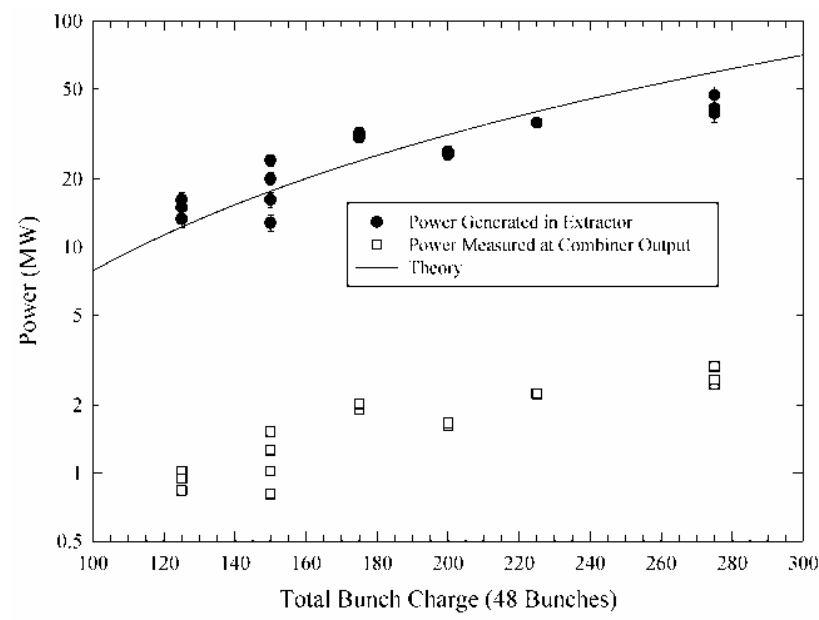

Figure 7: Power generated in the $21 \mathrm{GHz}$ ceramic based power extractor for various values of the total beam charge (48 bunches per pulse).

\section{CONCLUSIONS}

A ceramic based rf power extractor generated almost $50 \mathrm{MW}$ of power at $21 \mathrm{GHz}$. Despite some experimental difficulties and fabrication errors, this value for the generated power matched quite well with the anticipated power level. The measured spectra correlate well with the spectra extracted from a theoretical model with simple reflections at the ends of the ceramic extractor.

\section{REFERENCES}

[1] D. Yu, D. Newsham, A. Smirnov, Proc. $10^{\text {th }}$ Adv. Accelerator Workshop, AIP 547, pp. 484-505, 2002.

[2] W. Gai and P. Schoessow, Nuclear Instruments and Methods A459, pp. 1-5, 2001. 